\title{
Correlation between circulating tumour DNA and metabolic tumour burden in metastatic melanoma patients
}

\author{
Ashleigh C. McEvoy ${ }^{1}$, Lydia Warburton², Zeyad Al-Ogaili ${ }^{3}$, Liesl Celliers ${ }^{3}$, Leslie Calapre ${ }^{1}$, Michelle R. Pereira ${ }^{1}$, \\ Muhammad A. Khattak ${ }^{1,4,5}$, Tarek M. Meniawy ${ }^{2,5}$, Michael Millward ${ }^{2,5}$, Melanie Ziman ${ }^{1,6}$ and Elin S. Gray , $^{1 *}$ (D)
}

\begin{abstract}
Background: Circulating tumour DNA (ctDNA) may serve as a measure of tumour burden and a useful tool for non-invasive monitoring of cancer. However, ctDNA is not always detectable in patients at time of diagnosis of metastatic disease. Therefore, there is a need to understand the correlation between ctDNA levels and the patients' overall metabolic tumour burden (MTB).

Methods: Thirty-two treatment naïve metastatic melanoma patients were included in the study. MTB and metabolic tumour volume (MTV) was measured by 18F-fluoro-D-glucose positron emission tomography/computed tomography (FDG PET/CT). Plasma ctDNA was quantified using droplet digital PCR (ddPCR).

Results: CtDNA was detected in 23 of 32 patients. Overall, a significant correlation was observed between ctDNA levels and MTB $(p<0.001)$. CtDNA was not detectable in patients with an MTB of $\leq 10$, defining this value as the lower limit of tumour burden that can be detected through ctDNA analysis by ddPCR.

Conclusions: We showed that ctDNA levels measured by ddPCR correlate with MTB in treatment naïve metastatic melanoma patients and observed a limit in tumour size for which ctDNA cannot be detected in blood. Nevertheless, our findings support the use of ctDNA as a non-invasive complementary modality to functional imaging for monitoring tumour burden.
\end{abstract}

Keywords: Circulating tumour DNA, ctDNA, Metabolic tumour burden, Tumour lesion glycolysis, Melanoma, Droplet digital PCR

\section{Background}

Melanoma is an aggressive form of skin cancer that is increasing in prevalence worldwide [1]. Metastatic melanoma is a highly aggressive and difficult to treat cancer, particularly when patients present with advanced-stage disease that is unresectable [2]. Recent advances in understanding the molecular mechanisms of melanoma oncogenesis and immune evasion have resulted in the introduction of BRAF and immune checkpoint inhibiting agents. These new treatments have improved the melanoma survival [3-6], with the greatest treatment benefit

\footnotetext{
* Correspondence: e.gray@ecu.edu.au

${ }^{1}$ School of Medical and Health Sciences, Edith Cowan University, 270

Joondalup Drive, Joondalup, WA 6027, Australia

${ }^{7}$ Centre for Opthalmology and Visual Science, University of Western Australia,

35 Stirling Highway, Crawley, Western Australia 6009, Australia

Full list of author information is available at the end of the article
}

observed when treatment is initiated at a lower disease burden [2, 7-9].

In patients with advanced stage melanoma, treatment decisions are based upon clinical and imaging findings. In recent years, positron emission tomography with 2-deoxy-2[fluorine-18] fluoro-D-glucose integrated with computed tomography (FDG PET/CT) has emerged as a powerful imaging tool for initial staging and evaluating treatment response in metastatic melanoma [10, 11]. ${ }^{18} \mathrm{~F}-\mathrm{FDG}$ is a radio labelled glucose analogue which reflects tumour metabolic activity. Commonly, FDG PET/ $\mathrm{CT}$ is used to determine tumour burden as it provides a high tumour-to-background intensity ratio which facilitates computer generated measurements of total body metabolic tumour volume (MTV) and total lesion

(c) The Author(s). 2018 Open Access This article is distributed under the terms of the Creative Commons Attribution 4.0 International License (http://creativecommons.org/licenses/by/4.0/), which permits unrestricted use, distribution, and reproduction in any medium, provided you give appropriate credit to the original author(s) and the source, provide a link to the Creative Commons license, and indicate if changes were made. The Creative Commons Public Domain Dedication waiver (http://creativecommons.org/publicdomain/zero/1.0/) applies to the data made available in this article, unless otherwise stated. 
glycolysis (TLG) from which metabolic tumour burden (MTB) can be quantitatively calculated $[12,13]$.

As a blood-based biomarker, circulating tumour DNA (ctDNA) offers a non-invasive and easily accessible method of providing a real-time "snap shot" of tumour burden. The level of ctDNA sensitivity however differs between tumour types, AJCC stages, mutant forms and between patients [14]. In AJCC stage IV melanoma patients, ctDNA has been recognised as a valuable biomarker for tumour genetic profiling, monitoring disease progression, response to therapy and as a predictor of clinical outcome [15-23]. Whilst, ctDNA has been detected in $73-89 \%$ of patients prior to therapy initiation $[15,16,19,24]$ and the absence of ctDNA in these patients has been suggested as a prognostic marker for a better disease outcome [19], the level of overall tumour burden at which ctDNA can be detected has not yet been quantified.

Numerous platforms are available for the detection of ctDNA, however the droplet digital PCR (ddPCR) platform has been shown to be the most sensitive, capable of detecting mutant DNA (such as BRAF V600E) at $0.001 \%$ frequency abundance $[25,26]$. The lower limit in tumour size that shed detectable amounts of ctDNA into the blood is however unclear, and it may vary between cancer types. This information is critical for clinical validation of the efficacy ctDNA as a non-invasive complimentary method to functional imaging for monitoring of tumour burden in melanoma patients.

Here we determined the levels of ctDNA measured by ddPCR targeting tumour specific mutations in a cohort of 32 treatment naive stage IV melanoma patients. We evaluated whether the presence of ctDNA was associated with progression free survival and whether ctDNA levels correlated with MTB measured by FDG PET/CT.

\section{Methods}

\section{Patient and sample collection}

We retrospectively selected 32 from a cohort of American Joint Committee on Cancer (AJCC) 7th Edition, stage IV melanoma patients enrolled in a multi-centre study from February 2013 to May 2017. Inclusion criteria incorporated patients whose plasma sample was collected within 8 weeks from an FDG PET/CT scan and before any systemic therapy (See Additional file 1 for a detailed list of timing from FDG PET/CT scan to collection of bloods). As part of the patients' routine diagnostic work out, BRAF mutation testing were completed prior to enrolment in the study. Additional mutational profiling for those patients that were considered $B R A F$ wild-type was conducted on tumour tissue using Next Generation Sequencing (NGS). Written informed consent was obtained from all patients under approved Human Research Ethics Committee protocols from
Edith Cowan University (No. 11543) and Sir Charles Gardner Hospital (No. 2013-246), Western Australia.

\section{${ }^{18} \mathrm{~F}$-labeled fluorodeoxyglucose positron emission tomography/computed tomography}

FDG PET/CT scans were performed on combined PET/ CT scanners at approved nuclear medicine centres in Perth, Western Australia. After a minimum fasting period of $6 \mathrm{~h}$, patients were injected with $5 \mathrm{MBq}$ pr. $\mathrm{kg}$ $\pm 10 \%$ of ${ }^{18} \mathrm{FDG}$ (minimum $200 \mathrm{MBq}$ and maximum $600 \mathrm{MBq}$ ). Positron emission tomography was performed on patients with serum glucose levels below $11 \mathrm{nmol} / \mathrm{l}$ at an acquisition time of $3 \mathrm{~min}$ per bed position. To determine anatomical location and for attenuation correction purposes, a whole-body low-dose computed tomography scan was performed. Details of the imaging cameras are outlined (see Additional file 2). After being used for routine clinical purposes, all images were reviewed retrospectively and independently by two experienced nuclear medicine physicians, blinded to the ctDNA analysis. The MTV is calculated from the maximum length of the lesion using CT images obtained from the FDG PET/CT imaging data and the TLG is calculated as MTV x mean SUV in the volume [12]. Analysis was conducted on a Siemens Syngo workstation (Siemens Healthcare GMbH) with each specialist independently reporting the global TLG (MTB) and the global MTV in $\mathrm{cm}^{3}$, as per Additional file 2. An average of the two scores provided by the nuclear medicine physicians was used for final analysis.

\section{Plasma sample preparation and DNA extraction}

Blood samples were collected from stage IV melanoma patients, prior to initiation of any systemic therapy, into EDTA vacutainer tubes and stored at $4{ }^{\circ} \mathrm{C}$. Plasma was separated within $24 \mathrm{~h}$ by centrifugation at $1600 \mathrm{~g}$ for $10 \mathrm{~min}$, followed by a second centrifugation at $2000 \mathrm{~g}$ for $10 \mathrm{~min}$, and then stored at $-80{ }^{\circ} \mathrm{C}$ until extraction. Cell free DNA (cfDNA) was isolated from between 1 to $5 \mathrm{~mL}$ of plasma using the QIAamp Circulating Nucleic Acid Kit (Qiagen) as per the manufacturer's instructions and eluted in $40 \mu \mathrm{l}$ AVE buffer (Qiagen) and stored at $-80{ }^{\circ} \mathrm{C}$ until ctDNA quantification.

\section{CtDNA quantification}

CtDNA was quantified by ddPCR as previously described $[16,27]$. Briefly, a PCR mixture containing $1 \mathrm{x}$ ddPCR supermix, primer/probe sets for BRAF [see Additional file 2] (custom synthesized by Life Technologies), NRAS (Bio-Rad) or KIT (Bio-Rad) and 5 or $8 \mathrm{uL}$ cfDNA were emulsified with droplet generation using the Automatic Droplet Generator (AutoDG, Bio-Rad). Amplifications were performed using cycling conditions previously described [16]. Droplets were analysed through a QX200 
droplet reader (Bio-Rad) and data was analysed using QuantaSoft analysis software (Bio-Rad). To ensure the accuracy of results, each sample was tested minimally in duplicate. For quantification, a minimum of 10,000 acceptable droplets per $20 \mathrm{uL}$ reaction was required.

\section{Statistical analysis}

Association between patient characteristics and ctDNA positivity or negativity was calculated by Fisher's exact tests or unpaired T-test where appropriate. To assess the inter-observer agreement, Pearson's correlation analysis was performed and an evaluation of bias and precision was performed using Bland Altman analysis [28]. The correlation between MTV and MTB and the number of mutated copies of ctDNA was evaluated using Pearson's correlation after log-transformation of both variables. Progression free survival (PFS) was calculated as the time from the date of initiating therapy to the date of first reported progressive disease (PD) or censored with stable disease at the most recent visit. Median PFS was calculated using the Kaplan-Meier method and compared using the log-rank test. Cox proportional hazards regression analyses were performed to examine association of ctDNA, and other variables with PFS. Multivariate Cox regression models were evaluated using a stepwise approach with bidirectional elimination to determine the best fit model. Confidence intervals were set at 0.05 and significance levels are reported as $P<0.05$. Statistical analyses were performed using Statistical Package for Social Sciences for Window version 22 (SPSS, Chicago, IL) and plotted using GraphPad Prism version 5 .

\section{Results}

\section{Cohort}

A total of 32 patients with stage IV metastatic cutaneous melanoma were included in the study. Patient characteristics are shown in Table 1. The median time between blood collection and PET/CT was 1.9 weeks (range: 0.1-8 weeks, see Additional file 1). Patients were treated with targeted therapy and/or immunotherapy (see Additional file 1). After a median follow-up of 64.4 weeks (95\% CI: 46.2-82.6 weeks), 11 patients progressed whereas a large proportion retained control of the disease.

\section{Evaluation of plasma ctDNA}

Of the 32 tumour samples analysed, at least one mutation was known to be present with $94 \%$ of the samples harbouring a mutation in BRAF V600. Other mutations detected in ctDNA included KIT L576P (patient 28) and NRAS Q61L (patient 32) (Fig. 1). CtDNA was detected in 23 patients with a median concentration of 38 copies $/ \mathrm{mL}$ of plasma (range: 1.6-52,440 copies/mL). The remaining 9
Table 1 Patient characteristics

\begin{tabular}{ll}
\hline & $\begin{array}{l}\text { Total } \\
(n=32)\end{array}$ \\
\hline Gender & $62.5(20)$ \\
Male & $37.5(12)$ \\
Female & \\
Age & $57(25-83)$ \\
Median years (range) & \\
Stage & $12.5(4)$ \\
M1a & $6.3(2)$ \\
M1b & $81.3(26)$ \\
M1c & \\
Performance status, ECOG & $65.6(21)$ \\
0 & $34.4(11)$ \\
1- 2 & \\
LDH & $34.4(11)$ \\
Not elevated & $21.9(7)$ \\
Elevated & $43.8(14)$ \\
Not available
\end{tabular}

Abbreviation: ECOG Eastern Cooperative Oncology Group, LDH Lactate dehydrogenase. * Significant difference $p<0.05$

patients had no detectable ctDNA. A comparison of patient characteristics was conducted between the ctDNA positive and ctDNA negative patients (Table 2). Significant differences were evident in sex $(p=0.05)$, with a higher proportion of males having detectable ctDNA than females, and M stage ( $p=0.03$ ), with M1c (patients with visceral metastases) more likely to have detectable ctDNA than M1a and M1b. When evaluating for PFS, patients with detectable ctDNA had a significantly shorter median PFS (39.14 weeks) than patients with undetectable ctDNA (102.29 weeks, $P=0.002$; Fig. 2).

\section{Inter-observer analysis of MTV and MTB}

MTV and MTB were assessable in all patients from PET/ CT scans. A good correlation $\left(R^{2}=0.978\right.$ and 0.998 respectively) was observed between the two independent scores (see Additional file 3), with Bland-Altman plots showing a good agreement between the two specialists, with all but one observation falling within $95 \% \mathrm{CI}$.

\section{Correlation between ctDNA copies / $\mathrm{mL}$ and MTV/MTB}

A significant correlation $\left(\mathrm{R}^{2}=0.7054, \quad P<0.0001\right)$ (Fig. 3a) was found between the copies of ctDNA per $\mathrm{mL}$ of plasma and the global TLG or MTB. Similarly, a weaker but none the less significant correlation was observed $\left(R^{2}=0.6949, P<0.0001\right)$ (Fig. $\left.3 b\right)$ between the copies of ctDNA per mL of plasma and the MTV. CtDNA was undetectable in all patients with an MTB value of $\leq 10$, which suggests that this is the lower limit 


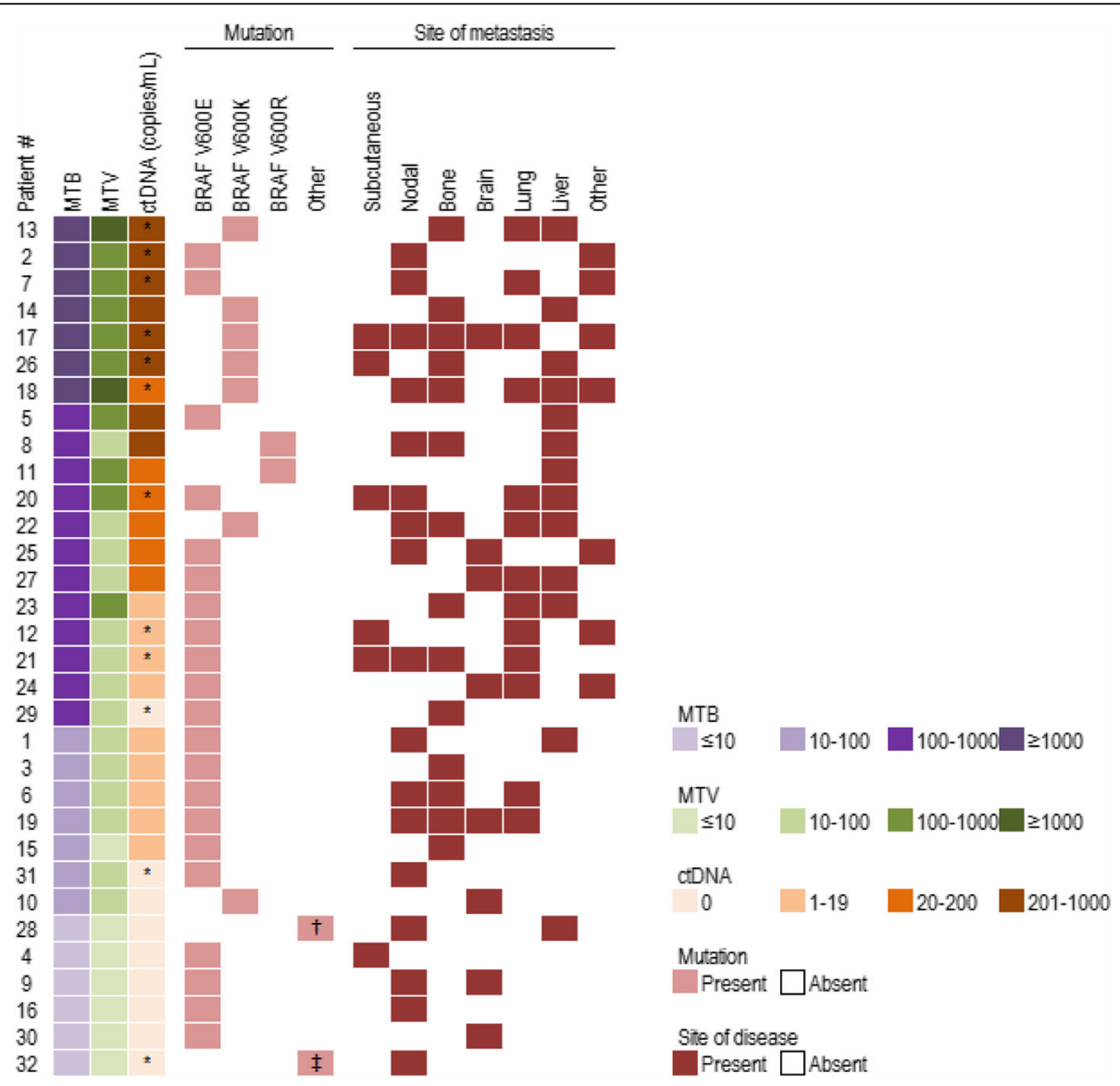

Fig. 1 Overview of disease burden and ctDNA for 32 patients with melanoma. Patients are ordered according to metabolic tumour burden (MTB). Other indications of tumour burden such as metabolic tumour volume (MTV) and site of metastasis are compared with circulating tumour DNA (ctDNA, copies/mL of plasma) levels and site of metastasis. CtDNA was measured by droplet digital PCR targeting the mutations indicated. *denotes cases for which cfDNA was extracted from $1 \mathrm{~mL}$ of plasma. †denotes KIT L576P, ‡denotes NRAS Q61L

of disease burden at which ctDNA can be detected. CtDNA was also undetectable in three cases with MTB > 10, namely patients 10, 29 and 31 (Fig. 1). CfDNA was isolated from $1 \mathrm{~mL}$ of plasma for patients 29 and 31 and patient 10 had a cerebellar lesion only brain metastasis which may reduce our capacity to detect ctDNA in these cases.

\section{Multivariate cox regression analyses of PFS}

Finally, in a univariate Cox regression analysis, detectable ctDNA, high MTB and M1c disease were found to be significantly associated with decreased PFS (Table 3). A multivariate Cox regression analysis revealed that these three variables do not independently predict PFS. Using a stepwise approach with bidirectional elimination to determine the best fit model, we found that ctDNA explains most of the differences in PFS with an adjusted $\mathrm{HR}=7.658$ (CI 95\% 1.762-33.283) (Table 3) after controlling for sex, age and ECOG status.

\section{Discussion}

Here we demonstrated a significant correlation between the ctDNA levels in plasma and MTB measured by PET/CT in metastatic melanoma patients. Moreover, we observed a threshold for detecting ctDNA at an MTB score of $\leq 10$. Finally, we confirm previous reports showing that undetectable ctDNA is associated with longer PFS.

With regards to patients with undetectable ctDNA, several cases are worth highlighting. In our study, one case (patient 4) with subcutaneous metastases only and noted to have low disease burden, was found negative for plasma ctDNA. Previously, subcutaneous metastases have been shown to be associated with low levels of ctDNA, despite extensive disease [22]. Patients 16, 31 and 32 had nodal metastases only and ctDNA was not detectable. Whilst Wong et al. [22] have shown that patients with nodal involvement often display high levels of ctDNA, we did not confirm this finding. It is worth noting that no other patients in our cohort had nodal 
Table 2 Comparisons of clinical characteristics between patients with and without ctDNA present $(N=32)$

\begin{tabular}{llll}
\hline & $\begin{array}{c}\text { ctDNA+ } \\
(n=23)\end{array}$ & $\begin{array}{l}\text { ctDNA- } \\
(n=9)\end{array}$ & $\begin{array}{c}P \\
\text { value }\end{array}$ \\
\hline $\begin{array}{l}\text { Gender } \\
\text { Male }\end{array}$ & $85.0(17)$ & $15.0(3)^{*}$ & $0.05^{*}$ \\
$\quad \begin{array}{l}\text { Female } \\
\text { Age }\end{array}$ & $50.0(6)$ & $50.0(6)$ & \\
$\quad$ Median years (range) & $58(25-78)$ & $62(34-83)$ & 0.24 \\
Stage & & & \\
$\quad$ M1a or b & $16.7(1)$ & $83.3(5)$ & $0.03^{*}$ \\
$\quad$ M1c & $84.6(22)$ & $15.4(4)$ & \\
Performance status, ECOG & & & 0.68 \\
$\quad$ O & $69.6(16)$ & $30.4(5)$ & \\
$>0.99$ & $63.6(7)$ & $36.4(4)$ & \\
LDH & & & \\
Not elevated & $63.6(7)$ & $36.4(4)$ & \\
Elevated & $85.7(6)$ & $14.3(1)$ & \\
Not available & $71.4(10)$ & $28.6(4)$ & \\
\hline
\end{tabular}

Abbreviation: ECOG Eastern Cooperative Oncology Group, LDH Lactate dehydrogenase. ${ }^{*}$ Significant difference $p<0.05$

disease alone, thus extrapolation of our results suggesting that ctDNA is undetectable in node only disease should be considered with caution. Moreover, this may be confounded by the fact that in two cases (patients 16 and 32) the MTB was below our threshold of 10. The third patient (patient 31) had an MTB of 33.49 but the lack of ctDNA could be attributed to the fact that cfDNA was extracted from only $1 \mathrm{ml}$ of plasma.

Undetectable ctDNA in patients 9,10 and 30 may be explained by the sites of their metastatic disease, nodal and brain (patient 9) and brain only (patient 10 and 30).
Previously, low or undetectable ctDNA levels have been observed in patients with brain metastases [22, 29, 30].

One significant outlier is patient 29 , where despite having bone metastases and a significant MTB, we were unable to detect ctDNA. Bone metastases have previously been associated with high levels of ctDNA [22]. In this case, cfDNA was also extracted from only $1 \mathrm{~mL}$ of plasma which is likely to have a significant impact on detection levels of ctDNA [31].

Remarkably patient 28 (with nodal and liver metastases) had undetectable ctDNA however, MTB was $\leq 10$. No obvious limitations were evident in this case, particularly with regards to the volume of plasma collected. Thus, we are confident that with our current assay sensitivities, we are not able to detect ctDNA in patients with a MTB value of $\leq 10$, regardless of disease site or mutation. Nevertheless, this observation needs to be corroborated in larger studies, taking in consideration diverse mutated genes and the site of metastases.

To our knowledge, this is the first study in melanoma that has directly compared the level of ctDNA with the exact MTB calculated from the sum of TLG for all evaluable lesions. Recently, Wong et al. [22] showed that ctDNA levels correlate with qualitative analysis of whole body MTV in metastatic melanoma patients. Similar to our findings, a strong correlation was observed between ctDNA levels and MTV $(r=0.61 ; P<0.001)$. Whilst it is difficult to make a direct comparison between this study and our own due to the different methodologies employed to detect ctDNA (digital PCR and targeted sequencing) and different reporting mechanisms of MTV (Wong reported results in $\mathrm{mL}$, whilst we reported results in $\mathrm{cm}^{3}$ ), it is interesting to note there is a remarkable difference in the median levels of plasma ctDNA reported by both studies. Wong et al. reported a median
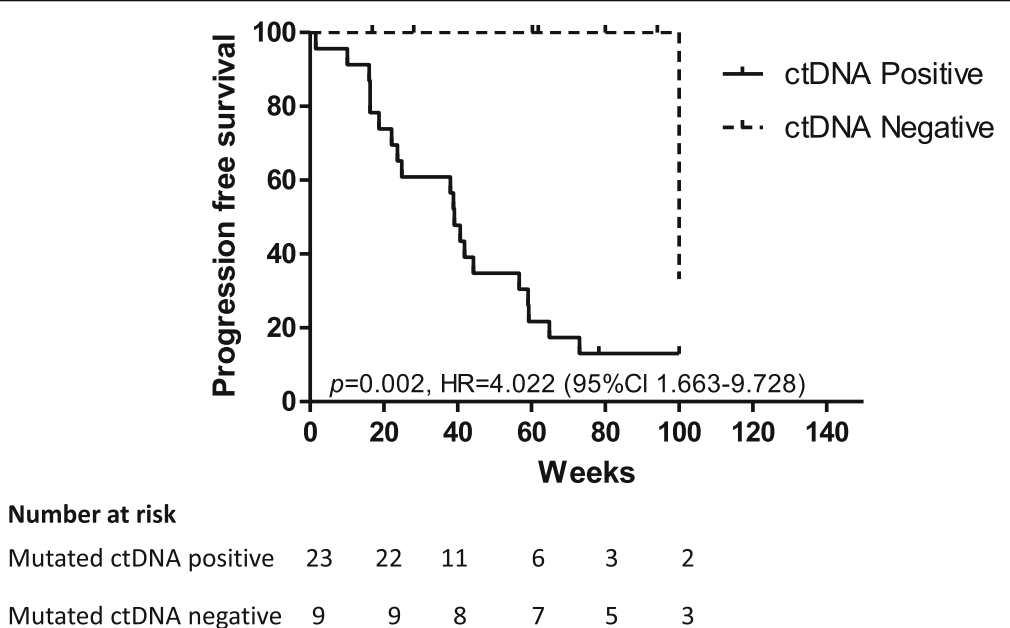

Fig. 2 Kaplan-Meier curve for progression free survival (PFS) according to the presence of circulating tumour DNA (ctDNA) in plasma. In patients with detectable ctDNA, a median PFS of 33.29 weeks was observed 

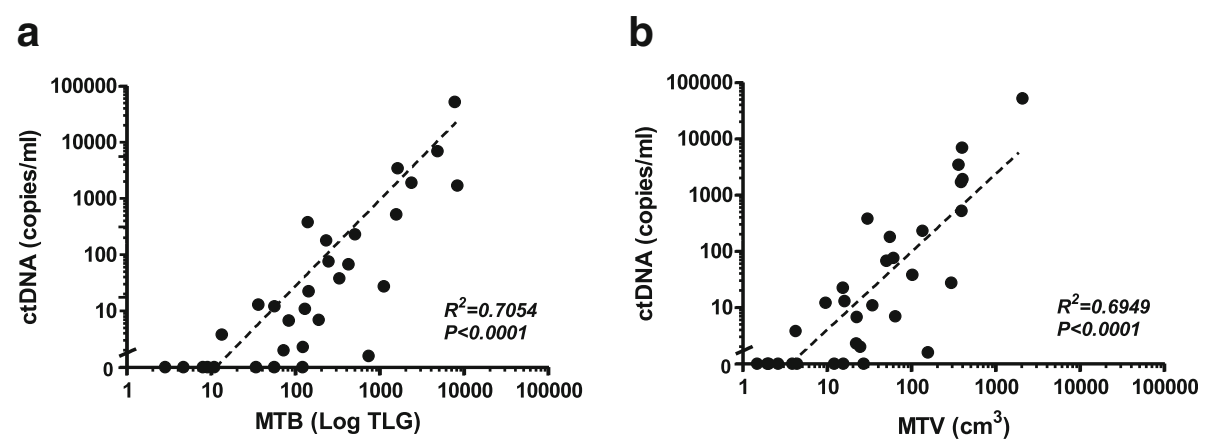

Fig. 3 Correlation between circulating tumour DNA (ctDNA) levels and disease burden. Correlation between ctDNA levels in copies/mL of plasma and MTB (a) or MTV (b) assessed by FDG-PET imaging. Analysis was performed by using Spearman rank correlation

ctDNA concentration of 1112 copies/mL of plasma (range 63-97,000) which is considerably higher than the copies/ $\mathrm{mL}$ of plasma that we reported, indicating an enrichment of patients with lower disease burden in our study. Finally, our measurements incorporated PET parameter TLG to define all measureable lesions combined with the metabolic activity of each tumour, thus measuring both tumour volume and aggressiveness. This approach provides a comprehensive score of not only of tumour burden but also of tumour activity and therefore, an overall perspective of the disease status of patient.

In line with our study, the presence of ctDNA has been directly correlated with patient survival and MTB in advanced stage non-small cell lung cancer (NSCLC) [12]. This study assessed allele frequency measured by NGS in 24 NSCLC patients and MTB (calculated from the sum of TLG for all evaluable lesions) and found a significant correlation $(P=0.001)$. The authors also reported a significantly shorter median overall survival (OS) in patients with detectable ctDNA compared to those with no detectable ctDNA. In our study, we have specifically chosen not to assess OS as this endpoint includes death from any cause, and thus is influenced by co-morbidities and access to systemic therapies over different periods of time. For this reason, our analysis focused on PFS, which was significantly shortened in patients with detectable ctDNA. Finally, multivariate Cox regression analyses demonstrated that ctDNA is not an independent variable but rather a reflection of other disease burden measurements such as MTB and disease stage.

There are a number of noteworthy limitations to our study, largely associated with its retrospective nature. Firstly, the timing of blood collections in accordance with PET/CT imaging was varied. In the Winther-Larsen and colleagues study [12], blood samples were collected at the time of inclusion into the study, and PET/CT imaging. This resulted in a median interval of 2 days between imaging and blood draw, which is considerably shorter than our median of 1.9 weeks. However, for both studies the timing between blood collection and imaging may be an important factor to consider. Given that ctDNA has a half-life of less than $2 \mathrm{~h}$ and have been shown to increase as new lesions become apparent [32], ideally blood draw should be conducted immediately after imaging to ensure that ctDNA detected is a true reflection of lesions identified in the image. Importantly, in all our cases, plasma was collected after imaging and prior to treatment. Secondly, PET/CT imaging was conducted at different institutions, which may have resulted in inter-institutional differences in quality control and scanning [33]; the use of multiple scanner models has been associated with variability in the

Table 3 Factors associated with PFS

\begin{tabular}{|c|c|c|c|c|c|c|c|}
\hline \multirow[t]{2}{*}{ Factor } & \multirow[t]{2}{*}{ Variable } & \multicolumn{3}{|c|}{ Univariate analysis } & \multicolumn{3}{|c|}{ Multivariate analysis } \\
\hline & & $p$-value & $\mathrm{HR}$ & $\mathrm{Cl}(95 \%)$ & $p$-value & $\mathrm{HR}$ & $\mathrm{Cl}(95 \%)$ \\
\hline ctDNA & positive/negative & $0.009^{*}$ & 6.973 & $1.612-30.165$ & $0.007^{*}$ & 7.658 & $1.762-33.283$ \\
\hline Age & Continuous & 0.619 & 0.992 & $0.961-1.024$ & 0.558 & 0.988 & $0.949-1.029$ \\
\hline Sex & Male vs. Female & 0.419 & 0.834 & $0.537-1.295$ & 0.548 & 1.184 & $0.682-2.058$ \\
\hline ECOG & $0-2$ & 0.747 & 1.127 & $0.544-2.337$ & 0.526 & 1.284 & $0.593-2.781$ \\
\hline MTB\$ & $>34$ vs. $\leq 34$ & $0.018^{*}$ & 5.845 & $1.356-25.206$ & & & \\
\hline Stage & M1a,b vs. M1c & $0.035^{*}$ & 1.454 & $0.218-0.945$ & & & \\
\hline
\end{tabular}

Abbreviations: ctDNA circulating tumour DNA, ECOG Eastern Cooperative Oncology Group, MTB Metabolic Tumour Burden, $H R$ hazard ratio, Cl confidence interval. *Statistically significant. \$Dichotomised at First Interquartile 
standard uptake of FDG readings [34]. We did not however observe any substantial deviation in the correlation between ctDNA and MTB due to scanner models (see Additional file 4). Finally, we acknowledge that this cohort is heavily biased for $B R A F \mathrm{~V} 600$ mutated cases, and future studies should address this across multiple mutations.

\section{Conclusions}

In conclusion, the significant correlation of ctDNA with MTB in treatment naïve metastatic melanoma patients in this study suggests that quantification of ctDNA between scans may provide a minimally invasive option with which to detect changes in disease burden in melanoma. We observed a limit in tumour size for which ctDNA can be detected in blood, suggesting that detection of ctDNA in patients with low disease burden necessitates further improvements in the technology to increase sensitivity.

\section{Additional files}

Additional file 1: Detailed list of timing from FDG PET/CT scan to collection of bloods and systemic treatments that patients received after FDG PET/CT and bloods. (XLSX $10 \mathrm{~kb}$ )

Additional file 2: Additional methodologies. (DOCX $16 \mathrm{~kb}$ ) Additional file 3: Concordance between nuclear physician MTB and MTV measurements. Correlations between the two analysts for MTB (A) and MTV (C). Bland-Altman plot for analysis of agreement for MTB (B) and MTV (D). (PPTX $64 \mathrm{~kb})$

Additional file 4: Correlation between MTB and ctDNA labelled according to the different scanner types. (PPTX $53 \mathrm{~kb}$ )

\section{Abbreviations}

AJCC: American Joint Committee on Cancer; cfDNA: Cell free DNA; ctDNA: Circulating tumour DNA; ddPCR: Droplet digital PCR; FDG PET/ CT: 18F-fluoro-D-glucose positron emission tomography/computed tomography; MTB: Metabolic tumour burden; MTV: Metabolic tumour volume; NGS: Next Generation Sequencing; NSCLC: Non small cell lung cancer; OS: Overall survival; PD: Progressive disease; PFS: Progression free survival; TLG: Total lesion glycolysis

\section{Acknowledgements}

The authors thank all the participants, patients and healthy volunteers, for their assistance with the study. We also would like to thank Johnny Lo for advice on statistical analysis.

\section{Funding}

This work was supported by a Western Australia Cancer Council grant (1100249) and a Perpetual Ramaciotti Foundation grant to EG, a National Health and Medical Research (NHMRC) grant (1119791) and a DoH WA grant $\mathrm{MZ}$ and a Spinnaker Health Research Foundation grant to MAK. AM has received financial support through an "Australian Government Research Training Program" scholarship and the Edith Cowan University "Inspiring Minds" scholarship. EG is supported by a fellowship from the Cancer Research Trust. Funding bodies were not involved in the design of the study, collection, analysis, and interpretation of data or in writing the manuscript.

\section{Availability of data and materials}

The datasets used and/or analysed during the current study are available from the corresponding author on reasonable request.

\section{Authors' contributions}

ACM designed the study and experiments, performed experiments, analysed data and wrote the manuscript. ZA and LCe assessed radiological imaging and provided expert advice. LW, MAK, TMM and MM recruited and clinically assessed patients and provided expert advice. LCa and MRP performed experiments and analysed data. MZ and ESG designed experiments, analysed data and supervised the study and writing of the manuscript. All authors contributed to the work presented in this study, reviewed, read and approved the final manuscript.

\section{Ethics approval and consent to participate}

This study was approved by the Human Ethics Committees at Edith Cowan University (No. 11543) and Sir Charles Gairdner Hospital (No.2013-246) and all patients provided written informed consent to participate.

\section{Consent for publication}

Not applicable.

\section{Competing interests}

ESG has received travel and accommodation sponsorship from Bio-Rad Laboratories.

\section{Publisher's Note}

Springer Nature remains neutral with regard to jurisdictional claims in published maps and institutional affiliations.

\section{Author details}

${ }^{1}$ School of Medical and Health Sciences, Edith Cowan University, 270 Joondalup Drive, Joondalup, WA 6027, Australia. ${ }^{2}$ Department of Medical Oncology, Sir Charles Gairdner Hospital, Hospital Avenue, Nedlands, WA 6009, Australia. ${ }^{3}$ Department of Molecular Imaging and Therapy Service, Fiona Stanley Hospital, Murdoch, WA 6150, Australia. ${ }^{4}$ Department of Medical Oncology, Fiona Stanley Hospital, 11 Robin Warren Drive, Murdoch, WA 6150, Australia. ${ }^{5}$ School of Medicine and Pharmacology, The University of Western Australia, 35 Stirling Highway, Crawley, WA 6009, Australia. ${ }^{6}$ School of Biomedical Sciences, University of Western Australia, 35 Stirling Highway, Crawley, WA 6009, Australia. ${ }^{7}$ Centre for Opthalmology and Visual Science, University of Western Australia, 35 Stirling Highway, Crawley, Western Australia 6009, Australia.

Received: 23 February 2018 Accepted: 26 June 2018

Published online: 09 July 2018

\section{References}

1. Miller KD, Siegel RL, Lin CC, Mariotto AB, Kramer JL, Rowland JH, Stein KD, Alteri R, Jemal A. Cancer treatment and survivorship statistics, 2016. Cancer. 2016:66(4):271-89

2. Luke JJ, Flaherty KT, Ribas A, Long GV. Targeted agents and immunotherapies: optimizing outcomes in melanoma. Nat Rev Clin Oncol. 2017;14:1-20.

3. Chapman PB, Hauschild A, Robert C, Haanen JB, Ascierto P, Larkin J, Dummer R, Garbe C, Testori A, Maio M. Improved survival with vemurafenib in melanoma with BRAF V600E mutation. NEJM. 2011; 364(26):2507-16.

4. Flaherty KT, Infante JR, Daud A, Gonzalez R, Kefford RF, Sosman J, Hamid O, Schuchter L, Cebon J, Ibrahim N. Combined BRAF and MEK inhibition in melanoma with BRAF V600 mutations. NEJM. 2012;367(18): 1694-703.

5. Long GV, Stroyakovskiy D, Gogas H, Levchenko E, de Braud F, Larkin J, Garbe C, Jouary T, Hauschild A, Grob JJ. Combined BRAF and MEK inhibition versus BRAF inhibition alone in melanoma. NEJM. 2014; 371(20):1877-88.

6. Robert C, Schachter J, Long GV, Arance A, Grob JJ, Mortier L, Daud A Carlino MS, McNeil C, Lotem M. Pembrolizumab versus ipilimumab in advanced melanoma. NEJM. 2015:372(26):2521-32.

7. Hodi FS, O'day SJ, McDermott DF, Weber RW, Sosman JA, Haanen JB, Gonzalez R, Robert C, Schadendorf D, Hassel JC. Improved survival with ipilimumab in patients with metastatic melanoma. NEJM. 2010;363:711-23.

8. Sosman JA, Kim KB, Schuchter L, Gonzalez R, Pavlick AC, Weber JS, McArthur GA, Hutson TE, Moschos SJ, Flaherty KT. Survival in BRAF 
V600-mutant advanced melanoma treated with vemurafenib. NEJM. 2012;366(8):707-14

9. McArthur GA, Larkin JM, Ascierto PA, Hsu JJ, Yan Y, Rooney IA, Koralek DO, Dréno B, Ribas A. Efficacy of cobimetinib (C) and vemurafenib $(V)$ in advanced BRAF-mutated melanoma patients (pts) with poor and favorable prognosis in the coBRIM phase III study. Proc Am Soc Clin Oncol. 2016;

10. Schmitt RJ, Kreidler SM, Glueck DH, Amaria RN, Gonzalez R, Lewis K, Bagrosky BM, Kwak JJ, Koo PJ. Correlation between early 18F-FDG PET/ CT response to BRAF and MEK inhibition and survival in patients with BRAF-mutant metastatic melanoma. Nucl Med Commun. 2016;37(2): 122-8.

11. Reinhardt MJ, Joe AY, Jaeger U, Huber A, Matthies A, Bucerius J, Roedel R, Strunk H, Bieber T, Biersack H-J. Diagnostic performance of whole body dual modality 18F-FDG PET/CT imaging for $\mathrm{N}$-and $\mathrm{M}$-staging of malignant melanoma: experience with 250 consecutive patients. J Clin Oncol. 2006; 24(7):1178-87.

12. Winther-Larsen A, Demuth C, Fledelius J, Madsen AT, Hjorthaug K, Meldgaard P, Sorensen BS. Correlation between circulating mutant DNA and metabolic tumour burden in advanced non-small cell lung cancer patients. Brit J Cancer. 2017:1-6.

13. Larson SM, Erdi Y, Akhurst T, Mazumdar M, Macapinlac HA, Finn RD, Casilla C, Fazzari M, Srivastava N, Yeung HW. Tumor treatment response based on visual and quantitative changes in global tumor glycolysis using PET-FDG imaging: the visual response score and the change in total lesion glycolysis. Clin Pos Imaging. 1999;2(3):159-71.

14. Schwarzenbach H, Hoon DS, Pantel K. Cell-free nucleic acids as biomarkers in cancer patients. Nat Rev Cancer. 2011;11(6):426.

15. Ascierto PA, Minor D, Ribas A, Lebbe C, O'Hagan A, Arya N, Guckert M, Schadendorf D, Kefford RF, Grob J-J. Phase II trial (BREAK-2) of the BRAF inhibitor dabrafenib (GSK2118436) in patients with metastatic melanoma. J Clin Oncol. 2013;31(26):3205-11

16. Gray ES, Rizos H, Reid AL, Boyd SC, Pereira MR, Lo J, Tembe V, Freeman J, Lee JH, Scolyer RA. Circulating tumor DNA to monitor treatment response and detect acquired resistance in patients with metastatic melanoma. Oncotarget. 2015:6(39):42008-18.

17. Sanmamed MF, Fernández-Landázuri S, Rodríguez C, Zárate R, Lozano MD, Zubiri L, Perez-Gracia JL, Martín-Algarra S, González A. Quantitative cell-free circulating BRAFV600E mutation analysis by use of droplet digital PCR in the follow-up of patients with melanoma being treated with BRAF inhibitors. Clin Chem. 2015;61(1):297-304.

18. Gonzalez-Cao M, Mayo-de-las-Casas C, Molina-Vila MA, De Mattos-Arruda L, Muñoz-Couselo E, Manzano JL, Cortes J, Berros JP, Drozdowskyj A Sanmamed M. BRAF mutation analysis in circulating free tumor DNA of melanoma patients treated with BRAF inhibitors. Melanoma Res. 2015;25(6): 486-95

19. Santiago-Walker A, Gagnon R, Mazumdar J, Casey M, Long GV, Schadendorf D, Flaherty K, Kefford R, Hauschild A, Hwu P. Correlation of BRAF mutation status in circulating-free DNA and tumor and association with clinical outcome across four BRAFi and MEKi clinical trials. Clin Cancer Res. 2015; https://doi.org/10.1158/1078-0432.CCR-15-0321.

20. Girotti MR, Gremel G, Lee R, Galvani E, Rothwell D, Viros A, Mandal AK, Lim KHJ, Saturno G, Furney SJ. Application of sequencing, liquid biopsies and patient-derived xenografts for personalized medicine in melanoma. Cancer Discov. 2015; https://doi.org/10.1158/2159-8290.CD-15-1336.

21. Lee J, Long G, Boyd S, Lo S, Menzies A, Tembe V, Guminski A, Jakrot V, Scolyer R, Mann G. Circulating tumour DNA predicts response to anti-PD1 antibodies in metastatic melanoma. Ann Oncol. 2017;28(5):1130-6.

22. Wong SQ, Raleigh JM, Callahan J, Vergara IA, Ftouni S, Hatzimihalis A, Colebatch AJ, Li J, Semple T, Doig K, et al. Circulating tumor DNA analysis and functional imaging provide complementary approaches for comprehensive disease monitoring in metastatic melanoma. Prec Oncol. 2017;(1):1-14.

23. McEvoy AC, Calapre L, Pereira MR, Giardina T, Robinson C, Khattak MA Meniawy TM, Pritchard AL, Hayward NK, Amanuel B, et al. Sensitive droplet digital PCR method for detection of TERT promoter mutations in cell free DNA from patients with metastatic melanoma. Oncotarget. 2017:8(45):78890-900

24. Lee R, Gremel G, Marshall A, Myers K, Fisher N, Dunn J, Dhomen N, Corrie P, Middleton $\mathrm{M}$, Lorigan $\mathrm{P}$, et al. Circulating tumor DNA predicts survival in patients with resected high risk stage II/III melanoma. Ann Oncol. 2017; https://doi.org/10.1093/annonc/mdx717.

25. Hindson BJ, Ness KD, Masquelier DA, Belgrader P, Heredia NJ, Makarewicz AJ, Bright IJ, Lucero MY, Hiddessen AL, Legler TC. High-throughput droplet digital PCR system for absolute quantitation of DNA copy number. Anal Chem. 2011:83(22):8604-10.

26. Reid AL, Freeman JB, Millward M, Ziman M, Gray ES. Detection of BRAFV600E and V600K in melanoma circulating tumour cells by droplet digital PCR. Clin Biochem. 2015;48(15):999-1002.

27. McEvoy A, Wood B, Ardakani N, Pereira MR, Pearce R, Cowell L, Robinson C, Grieu-lacopetta F, Spicer A, Amanuel B, et al. Droplet digital PCR for mutation detection in foramalin fixed parafin embedded melanoma tissues: a comparison with sanger sequencing and pyrosequencing. J Mol Diagn. 2017;20(2):240-52.

28. Bland JM, Altman DG. Statistical methods for assessing agreement between two methods of clinical measurement. Lancet. 1986;1(8476):307.

29. Bettegowda C, Sausen M, Leary RJ, Kinde I, Wang Y, Agrawal N, Bartlett BR, Wang $\mathrm{H}$, Luber B, Alani RM. Detection of circulating tumor DNA in early-and late-stage human malignancies. Sci Transl Med. 2014;6(224):224ra224.

30. De Mattos-Arruda L, Mayor R, Ng CK, Weigelt B, Martínez-Ricarte F, Torrejon D, Oliveira M, Arias A, Raventos C, Tang J. Cerebrospinal fluid-derived circulating tumour DNA better represents the genomic alterations of brain tumours than plasma. Nat Comms. 2015;6:8839.

31. Sherwood JL, Corcoran C, Brown H, Sharpe AD, Musilova M, Kohlmann A Optimised pre-analytical methods improve KRAS mutation detection in circulating tumour DNA (ctDNA) from patients with non-small cell lung cancer (NSCLC). PLoS One. 2016;11(2):e0150197.

32. Diehl F, Schmidt K, Choti MA, Romans K, Goodman S, Li M, Thornton K, Agrawal N, Sokoll L, Szabo SA. Circulating mutant DNA to assess tumor dynamics. Nat Med. 2008;14(9):985-90.

33. Bai B, Bading J, Conti PS. Tumor quantification in clinical positron emission tomography. Theranostics. 2013;3(10):787.

34. Fahey FH, Kinahan PE, Doot RK, Kocak M, Thurston H, Poussaint TY. Variability in PET quantitation within a multicenter consortium. Med Phys. 2010;37(7):3660-6.

\section{Ready to submit your research? Choose BMC and benefit from:}

- fast, convenient online submission

- thorough peer review by experienced researchers in your field

- rapid publication on acceptance

- support for research data, including large and complex data types

- gold Open Access which fosters wider collaboration and increased citations

- maximum visibility for your research: over $100 \mathrm{M}$ website views per year

At BMC, research is always in progress.

Learn more biomedcentral.com/submissions 